\section{Tendencias en las políticas de atención a la dependencia de ancianos y sus reformas}

\author{
Trends and reforms in long-term care policies \\ for the elderly
}

\section{Tendências e reformas nas políticas de atenção aos idosos com dependência}

\author{
1 Departamento de Economía, \\ Métodos Cuantitativos \\ e Historia Económica, \\ Universidad Pablo de \\ Olavide, Sevilla, España. \\ Correspondencia \\ M. Matus-López \\ Departamento de Economía, \\ Métodos Cuantitativos \\ e Historia Económica \\ Universidad Pablo de \\ Olavide. \\ Ctra. de Utrera, km. 1, Sevilla \\ / España-41013. \\ mmatlop@upo.es
}

\begin{abstract}
One of the main public health concerns in medium and high-income countries is how to deal with problems of functional dependency of a growing number of elderly individuals. This study aimed to identify converging issues in 30 countries with formal long-term care systems. A systematic review included articles, studies, and comparative international reports pub lished from 2010 to 2015. The results show three trends in the design and development of these policies: (a) focus on the oldest or most dependent elderly, (b) expansion of financing based on individual contribution, and (c) promotion of home care and financial benefits for care in specialized centers (nursing homes and similar establishments). All three have positive effects on cost containment, despite limited evidence of impacts on people's health.
\end{abstract}

Public Health Policy; Long-Term Care; Demographic Aging; Healthcare Financing
Mauricio Matus-López 1

\section{Resumen}

Una de las mayores preocupaciones respecto a la salud pública de los países de renta media y alta es cómo enfrentar los problemas de dependencia funcional de un número cada vez mayor de personas en la tercera edad. El objetivo de este estudio fue identificar aspectos de convergencia de treinta países que poseen un sistema formal de cuidados. Para eso se realizó una revisión sistemática de artículos, estudios e informes comparativos internacionales, publicados entre 2010 y 2015. Los resultados muestran tres tendencias en la concepción y desarrollo de esas políticas: (a) concentrarse en las personas ancianas o en los ancianos más dependientes, (b) ampliación de la base de financiación en función a la contribución individual, (c) promoción de cuidados en casa y beneficios financieros para la atención en centros especializados (asilos, albergues o similares). Todos ellos tienen efectos positivos sobre la contención de costos, pese a que haya pocas evidencias de impactos sobre la salud de las personas.

Políticas Públicas de Salud; Cuidados a Largo Plazo; Envejecimiento de la Población; Financiación de la Atención de la Salud 


\section{Introducción}

Durante los últimos 25 años, la tasa de crecimiento de la población de 65 y más años de edad ha sido más del doble que el total mundial, por lo que se espera que los 596 millones que son en la actualidad sobrepasen el umbral de los 1.000 millones antes de dos décadas. En la actualidad, en los países de alto ingreso esta población alcanza al $16,4 \%$ y se espera que aumente hasta el $25,4 \%$ en 2050 1. Pero el mayor crecimiento en los próximos años ocurrirá en los países de ingresos medios ${ }^{2}$. Por ejemplo, en la Unión Europea se estima que los mayores de 65 años aumentarán un 50,8\% de aquí a 2050, mientras que en América Latina lo harán un 213\% en el mismo periodo 1 .

El envejecimiento poblacional va asociado a un natural desgaste de las funciones básicas del ser humano. Aunque existe discusión acerca de qué proporción de los años de vida ganados por el aumento de la esperanza de vida se vivirán en condiciones de dependencia, lo cierto es que el incremento en el número de personas mayores producirá tensiones en los sistemas de salud y sociales de los países de menos ingresos.

En la actualidad sólo una treintena de países en el mundo cuentan con sistemas formales de cuidados para las personas dependientes o cuidados de larga duración en su traducción al inglés (long-term care). Más allá de la particularidad de cada uno, éstos se pueden definir como la organización y provisión de un amplio rango de servicios de salud y sociales destinados a las personas que encuentran limitada su capacidad para desarrollar actividades de la vida diaria (bañarse, alimentarse o vestirse, entre otras) 3,4 .

Los antecedentes más antiguos de implementación de este tipo de políticas se encuentran en aquellos países donde la construcción del estado de bienestar se desarrolló bajo una amplia definición de derechos sociales, como en Holanda, Noruega o Dinamarca. No obstante, fue en la década de los años noventa cuando la mayoría de los países desarrollaron los suyos, como Alemania, Austria o Francia 5. Entre finales de aquella década y lo que va de este siglo, se han abierto amplios debates en muchos países como Japón, Estados Unidos y el Reino Unido, se han realizado profundas reformas en prácticamente todos los modelos existentes y se han desarrollado algunos nuevos como en España, Portugal o Corea del Sur.

Durante el siglo pasado, la clasificación más tradicional de los modelos de cuidados era tipo estanco: los países nórdicos con modelos universalistas; los de Europa continental de inspiración Bismarck; los mediterráneos con tipo asis- tencia social, y por último, aquellos sin sistemas formales.

Esta fotografía ha cambiado. La última ola de reformas, y el acuciante impacto de la crisis económica sobre los presupuestos fiscales de los países ricos, ha supuesto cierta convergencia de los modelos 6. De esta manera, la categorización tipo estanco parece haber dado paso a una descripción con base en la gradualidad, que no permite clasificaciones discretas o bien sólo identifica dos grupos: uno compuesto por pocos países con sistemas generosos y otro por la gran mayoría de países con menores diferencias en sus indicadores 7 .

En el presente trabajo se ha desarrollado una revisión exhaustiva de la bibliografía de los últimos cinco años (2010-2015) en los que se compararon distintos modelos de cuidados de la dependencia. El objetivo fue identificar tendencias en la cobertura, financiación y servicios ofrecidos que puedan servir de experiencia para aquellos países de ingresos medios que en los próximos años enfrentarán esta problemática y que, como señala la Organización Mundial de la Salud (OMS) 2, ineludiblemente deberán adoptar políticas similares.

\section{Método}

Las fuentes de información y técnicas incluidas fueron tres: (a) análisis documental de los sistemas nacionales de cuidados y sus reformas, (b) análisis documental de informes comparativos de organismos internacionales y (c) revisión de la literatura sobre los últimos procesos de reforma y análisis comparativos entre políticas de cuidados. En todos los casos, la búsqueda se limitó a la información más reciente.

El análisis de los modelos por separado se llevó a cabo fundamentalmente a través de la revisión de los 22 informes nacionales del proyecto Assessing Needs of Care in European Nations para el periodo 2010-2012 8, la explotación de los últimos resultados de 2014 del Mutual Information System on Social Protection para 32 países 9, las páginas oficiales de los distintos gobiernos y el informe de la Comisión Dilnot para la reforma del sistema británico de 201110.

El análisis de informes institucionales se centró en el último Informe de Salud Global de la OMS 2 y los análisis especializados más recientes de la Organización para la Cooperación y Desarrollo Económico (OECD) 4,11,12 y de la Comisión Europea (EC) 3,6,13.

Por último, la revisión bibliográfica se circunscribió al periodo comprendido entre enero de 2010 y enero de 2015. Se llevó a cabo bajo 
cuatro criterios de búsqueda de palabras claves en inglés y español: Long-term care \& lessons (dependencia y lecciones); Long-term care \& reforms (dependencia y cuidados); Long-term care \& policies (dependencia y políticas); Longterm care \& financing (dependencia y financiación). Las bases de datos utilizadas fueron: MEDLINE/PubMed, Scopus, Web of Science, JSTOR y Proquest.

Se revisaron un total de 1.084 documentos académicos que fueron sometidos a un proceso de selección con base en dos criterios: representar al mayor número de países con sistemas formales de cuidados y que aborden sus análisis desde la salud pública y el análisis económico. Con esto, se obtuvieron 17 artículos académicos publicados en revistas de alto impacto y 2 libros de editoriales de prestigio internacional que se organizaron en torno a tres ejes de análisis: acceso y cobertura del sistema; fuentes de financiación; servicios de atención a la dependencia.

\section{Resultados}

El criterio de una clasificación en continuo, más que en compartimentos estanco, es el que subyace en los trabajos que analizan la convergencia de los modelos. La revisión bibliográfica llevada a cabo en este estudio permite identificar al menos tres grandes ejes de convergencia: la definición del acceso, la forma de financiación y la canasta de servicios y prestaciones económicas.

\section{Hacia la cobertura universal focalizada}

Una de las primeras y más importantes decisiones que enfrentaron los modelos de cuidados de la dependencia fue definir el acceso como universalista o focalizado, y en este último caso, si iba a estar delimitado por el nivel de ingresos, por el patrimonio o por la severidad 14. Un estudio comparativo internacional 15 realizado después de la última ola de reformas, muestra que casi todos cuentan con una declaración de acceso universal. Sólo Italia, Estados Unidos y Reino Unido fueron catalogados como focalizados por ingreso. Los tres han sido duramente criticados en varios estudios.

El caso italiano puede ser discutido en su definición, pero muy poco en su diagnóstico. La mayor parte de los trabajos descriptivos señalan que el modelo necesita ser reformado desde hace décadas. Es un modelo desestructurado, con profundas desigualdades y baja coordinación administrativa. En la práctica las prestaciones económicas se gestionan a nivel nacional y los servicios a nivel local, creando fuertes asimetrías territoriales de acceso 16,17.

Estados Unidos acaba de someter a debate parlamentario el conocido como Obama Care o Community Living Assistance Services an Supports Act, que pretendía ampliar la cobertura en salud al menos a una parte de los 40 millones de personas sin seguro y particularmente ofrecer algún tipo de servicios a cerca de 5 millones de personas dependientes sin ningún tipo de atención 18. Lamentablemente, el Congreso rechazó este proyecto en 2013, pero dejó al descubierto las enormes necesidades de cuidados de parte importante de la población.

De los tres señalados, el modelo británico ha sido uno de los más estudiados, de los más criticados y recientemente, sometido a reforma. $\mathrm{Su}$ diagnóstico como un sistema precario, fragmentado e insolidario 19,20, llevó al Gobierno a encargar a una comisión la elaboración de propuestas de mejora y ampliación de la cobertura 10 . Actualmente, éstas se encuentran en proceso de discusión e implementación.

Aunque existe abundante evidencia que confluye hacia la declaración de acceso universal, esta universalidad tiene algunos componentes que permiten focalizar los esfuerzos hacia aquellos más necesitados. El primero de ellos es el mecanismo previo de definir quiénes son dependientes y además, desde qué nivel de dependencia se considerarán beneficiarios. Por ejemplo, Italia utiliza el índice de Katz, Portugal se basa en éste y el Barthel y otros como Alemania, Francia, Austria y España definen sus propios baremos. Pese a que es posible establecer cierta equivalencia entre cada medición, la subjetividad se materializa a la hora de establecer desde qué punto, valor o categoría de cada escala se considera a una persona dependiente como beneficiaria. Además, la progresividad y la generosidad de servicios y prestaciones de acuerdo al nivel de dependencia se define discrecionalmente desde la Administración, lo que permite destinar más o menos recursos a los más graves.

Esta mezcla entre acceso universal y focalización es lo que se denomina universalismo focalizado 21. El concepto es en definitiva un intento por mantener un modelo generoso y abierto a toda la población, pero con un grado de flexibilidad que permita realizar ajustes en caso de problemas de financiación y que estos afecten menos a las personas con grados más severos de dependencia.

\section{Ampliación de la base de financiación}

En la actualidad existen básicamente cuatro fuentes de financiación de los servicios para el 
cuidado de la dependencia: los impuestos generales (directos e indirectos), las contribuciones (del empleador, del trabajador y del pensionista), el aseguramiento privado y los copagos.

La mayor parte de los países estudiados financian sus sistemas públicos con impuestos generales (nacionales, regionales o locales) 4,15. Las principales excepciones son Japón y Alemania. En el primer caso, los recursos provienen en partes iguales de impuestos y contribuciones. Estas últimas son pagadas escalonadamente por edad. Aquellos de 40 a 65 años cotizan 2/3 del total y los mayores de 65, 1/3 restante. Alemania, por su parte estableció en 1995 un seguro público obligatorio que representa la totalidad de la financiación del sistema y en el que contribuyen trabajadores y empleadores en la misma proporción (así como autoempleados y pensionistas) 22.

Aunque la evidencia es rotunda a favor de los impuestos y las contribuciones, lo cierto es que en los últimos años, nuevas formas de financiación están comenzando a implementarse o a aumentar su importancia en el sistema con el objetivo de ampliar la base de recursos económicos y hacer sostenible el gasto público.

El problema de la financiación y la sostenibilidad fiscal es señalado como el de mayor prioridad para los responsables de los sistemas de atención a la dependencia en prácticamente todos los países consultados 11 .

El motivo es que el crecimiento del gasto en dependencia y sus proyecciones para los próximos años son alarmantes. Entre 2005 y 2011 los presupuestos en dependencia de los países de la OECD aumentaron a una tasa anual de 4,8\% mientras que sus economías lo hicieron apenas a un 1,3\%. Esta tendencia llevaría prácticamente a duplicar el gasto de aquí a 2060 4,12 e incluso podría hacerlo una década antes si el escenario es negativo 21. Sólo en la Unión Europea esto llevaría a que el presupuesto superara el 3\% del PIB en 2050 13.

Es por ello que en los primeros diez años de la década, más del $60 \%$ de los países con sistemas de cuidados para la dependencia modificaron sus estructuras de financiación 11. Las medidas tuvieron como objetivo aumentar la capacidad recaudatoria, aunque también reducir el gasto y mejorar la eficiencia en su uso 15,19,23.

En este contexto, han vuelto a ganar peso las posturas en favor de ampliar la participación de los copagos y sobre todo de impulsar el aseguramiento individual 21.

Los copagos están presentes en más de la mitad de los modelos existentes, pero su alcance sigue siendo limitado. Lo que ocurre es que su función como des-animador de la demanda espuria puede ser efectiva en algunos casos, pero como fuente de financiación es contraproducente. El esfuerzo financiero individual puede generar incentivos para un aplazamiento temporal de la demanda hacia un futuro de mayor complejidad y costo.

En cuanto a los seguros privados individuales, siguen presentando demasiados fallos para constituirse como una alternativa para el problema en su conjunto. El motivo es que las particularidades del riesgo catastrófico en el largo plazo, como es el caso de la dependencia, es proclive a generar fallos de mercado como asimetrías de información, la selección adversa y el riesgo moral. De esta manera, las aseguradoras elevan las primas y reducen las coberturas frente a la incertidumbre, lo que deriva en bajos incentivos a la contratación de éstos 24,25,26. Por ello, su utilización se ha recomendado principalmente como un mecanismo suplementario sobre un nivel básico de seguro social público 27 . Otra posible solución, en línea con los resultados de la Comisión Dilnot 10, es la que implementará el gobierno británico a partir del próximo año. Esta consiste en establecer un techo de gasto individual por sobre el cuál es Estado se hace responsable. Así, el gasto esperado deja de ser incierto para las aseguradoras, lo que podría abaratar las primas. No obstante, sus consecuencias y la factibilidad de incorporar esta financiación no se conocerán hasta entonces.

\section{Canastas de servicios y prestaciones económicas}

Los servicios para la atención a la dependencia se pueden agrupar fundamentalmente en dos: asistencia en el domicilio y atención en residencias. Se entiende como asistencia en domicilio, los servicios que permiten a las personas mayores dependientes continuar viviendo en sus domicilios. En cambio, la atención en residencias se refiere a los servicios que proveen conjuntamente alojamiento/estancia y cuidados de larga duración, como centros geriátricos, albergues especializados, nursing homes, etc. (se excluyen hospitales). A esto hay que agregar las prestaciones económicas destinadas al cuidado en el entorno familiar o a la contratación de servicios externos 4,11 .

Las tendencias que han marcado los últimos años han sido un balance a favor de la atención domiciliaria por sobre la atención residencial y la incorporación de prestaciones económicas en la mayoría de modelos 14,15,19,21,23.

En los últimos diez años, 16 países de la OECD realizaron reformas sobre el uso de servicios, de manera que entre 2001 y 2011 la proporción de atención domiciliaria sobre la atención formal 
(domiciliaria y residencial) pasó del 58\% al 63,3\% 4,11. Así, al comienzo de la presente década prácticamente todos los países con sistemas de cuidados tenían mayor número de personas recibiendo atención domiciliaria que residencial 28, aunque con un importante rango de valores. Por ejemplo, entre los países con mayores tasas de cobertura como Holanda o los nórdicos, cerca del $20 \%$ de la población de 65 y más años de edad recibía este servicio, mientras que en los países con menor cobertura, como Italia o Europa del Este, esta proporción apenas llegaba al 5\% 7 .

El argumento más socorrido para sustentar este cambio de orientación hacia la atención domiciliaria se basa en favorecer la libertad de elección de las personas dependientes, quienes responden que, con independencia de la edad y el sexo, prefieren envejecer en sus hogares 29 . No obstante, no se puede obviar el posible incentivo de las administraciones para contener el gasto público, ya que el costo de una plaza en residencia puede duplicar el de la atención domiciliaria 15. De hecho, existe evidencia acerca de una proporción mayor de atención domiciliaria en gobiernos locales con bajos ingresos que en localidades de altos ingresos ${ }^{30}$. Además, esto se torna relevante puesto que los que adoptan esta preferencia pro-domiciliaria someten a una mayor tensión los procesos de coordinación de los distintos niveles de servicios de salud 31 .

El segundo cambio tiene que ver con la incorporación de prestaciones económicas, denominadas cash-for-care 17 . Hace quince años, esta opción estaba presente en muy pocos países y su uso era más bien limitado, con la excepción de Italia, en el que la Indennità di accompagnamento había sido creada en 1980. Al finalizar la década pasada más de veinte países cuentan con algún tipo de prestación económica en sus sistemas de cuidado de la dependencia 11 .

Pero no existe un único tipo de prestación. Podrían al menos identificarse dos modalidades 6 , aunque en algunos países la clasificación en una u otra no sea del todo clara. La primera prestación está financieramente más dotada, es pagada al dependiente, tiene algunas restricciones de contratación y se mantiene el control sobre la formación de los cuidadores. El segundo tipo de prestación consiste en un pago de menor cuantía, dirigido a la persona cuidadora o la familia y en el que se tiene escaso o nulo control sobre su uso.

Así, el monto y el control del gasto realizado con esta dotación definen si se trata de un salario que promueve la atención formal o es una ayuda que consolida la atención informal. Los ejemplos más paradigmáticos en este continuo son los de los países nórdicos en la primera cate- goría de prestaciones, donde tienen un alcance controlado y limitado a la contratación formal y en la segunda categoría los países mediterráneos como Italia o España, pero también Austria, donde su utilización es amplia y favorece la consolidación de los cuidados informales. En un lugar intermedio se podría ubicar el caso de Francia, puesto que la prestación está sometida a ciertos controles al igual que los cuidadores, pero su utilización es mucho más extensa que en los países nórdicos 17 .

\section{Discusión}

Existe abundante evidencia que confirma algunas tendencias comunes en las políticas de cuidados para la dependencia. La primera de ellas es el denominado universalismo focalizado. Este consiste en definir el acceso al sistema como universal, independiente del nivel de ingreso o patrimonio de los beneficiarios, pero prioriza sobre las personas con mayores necesidades de salud. Este concepto está presente en casi todas las reformas llevadas a cabo en la última década y permite mantener la misma declaración de derechos individuales y sociales, pero distribuir la gama de recursos y su costo de forma prioritaria a las necesidades y a las limitaciones presupuestarias. Por una parte, se mantienen los derechos, pero por otra parte, se ajusta la dotación de los servicios.

Una segunda característica en común son los esfuerzos por ampliar la base de financiación de los sistemas. Actualmente, el grueso de los recursos provienen de impuestos generales y/o de las contribuciones de las personas ocupadas y pensionistas. No obstante, existen esfuerzos explícitos por impulsar el aseguramiento individual privado como una herramienta suplementaria a una base universal básica. Los resultados no son demasiado alentadores, ya que los fallos de mercado elevan las primas y reducen las coberturas. En esta dirección avanza la reforma británica que busca reducir la incertidumbre con un tope máximo de gasto individual por sobre el que el Estado de hace responsable. Así, la parte a pagar puede estar suscrita a un seguro de cobertura definida. En el mismo sentido de aumentar la aportación individual, los modelos han incorporado o consolidado el establecimiento de copagos. Este mecanismo es controvertido puesto que puede derivar en aplazamientos de la consulta en el tiempo, aumentando la complejidad y costo de la atención en el futuro, más aún cuando el sistema público actúa como última red de asistencia.

En tercer lugar, en los últimos diez años la atención residencial ha disminuido en términos 
relativos, dando mayor importancia a la atención domiciliaria y a un variado espectro de prestaciones económicas. De esta forma, el mix de servicios parece favorecer en envejecimiento en los hogares a través de las prestaciones económicas y de los servicios cuidados en el domicilio del beneficiario. Ambos mecanismos resultan menos caros que las plazas residenciales y parecen ser preferidos por los beneficiarios, de manera que pueden existir incentivos desde ambas partes para seguir impulsándolos.

En resumen, las tres tendencias no son neutras desde el punto de vista financiero. Voluntaria o involuntariamente todas coinciden en un efecto sobre la sostenibilidad económica a través de la contención del gasto o la ampliación de las fuentes de financiación. Esto puede tener efectos negativos sobre el resultado en salud de la persona beneficiaria. De poco sirve ajustar el costo en los servicios o en la financiación, si se traduce en un empeoramiento de la salud futura e intervenciones más complejas y caras en periodos siguientes. No obstante, actualmente se carecen de suficientes estudios que confirmen o rechacen esta afirmación.

Si efectivamente estas medidas son eficientes sanitaria y económicamente, serán de particular utilidad para muchos países. Como se comentó al inicio de este trabajo, en los próximos años serán los países de ingreso medio y medio-alto, la mayor parte del mundo, los que deberán buscar soluciones de salud pública al problema de la dependencia. Desde este punto de vista, las reformas o tendencias de los países ricos a ajustar sus sistemas de protección hacia modelos de menor gasto, puede transformarse en una valiosa fuente de información a la hora en que los países de menos ingresos diseñen sus propios modelos.

\section{Resumo}

Uma das maiores preocupações com a saúde pública dos países de renda média e alta é como enfrentar os problemas de dependência funcional de um número cada vez maior de pessoas mais velhas. O objetivo deste estudo foi identificar aspectos de convergência de trinta países que possuem um sistema formal de cuidados. Para isso realizou-se uma revisão sistemática de artigos, estudos e informes comparativos internacionais publicados entre 2010 e 2015. Os resultados mostram três tendências na concepção e desenvolvimento dessas políticas: (a) concentrar-se nas pessoas mais velhas ou nos idosos mais dependentes, (b) am- pliação da base de financiamento com base em contribuição individual, (c) promoção de cuidados em casa e benefícios financeiros para atendimento en centros especializados (asilos, albergues ou similares). Todos os três têm efeitos positivos sobre a contenção de custos, embora haja pouca evidência de impactos sobre a saúde das pessoas.

Políticas Públicas de Saúde; Assistência de Longa Duração; Envelhecimento da População;

Financiamento da Assistência à Saúde 


\section{Referencias}

1. World Bank. WB Statistical Information System http://data.worldbank.org/data-catalog/healthnutrition-and-population-statistics (accedido el 05/Feb/2015).

2. World Health Organization. Global brief for world health day. Geneva: World Health Organization; 2012.

3. Economic Policy Committee. The 2012 ageing report: economic and budgetary projections for the 27 EU member states. Brussels: European Commission; 2012.

4. Organization for Economic Co-operation and Development. Health at a glance 2014. Paris: Organization for Economic Co-operation and Development; 2014.

5. Matus M, Rodríguez P. Presiones de oferta y demanda sobre políticas formales de cuidados en Latinoamérica. Revista del CLAD Reforma y Democracia 2014; 60:103-30.

6. Bettio F, Verashchagina A. Long-term care for the elderly: provisions and providers in 33 European countries. Luxembourg: European Commission; 2010.

7. Carrera F, Pavolini E, Ranci C, Sabbatini A. Longterm care systems in comparative perspective: care needs, informal and formal coverage, and social impacts in European countries. In: Ranci C, Pavolini E, editors. Reforms in long-term care policies in Europe. New York: Springer; 2013. p. 23-52.

8. European Commission. Assessing needs of care in European nations, ANCIEN Project. http://www. ancien-longtermcare.eu/home (accedido el 10/ Dic/2014)

9. European Commission. Mutual information system on social protection. http://ec.europa.eu/so $\mathrm{cial} / \mathrm{main}$.jsp? catId=815\&langId $=$ en (accedido el 15/Ene/2015).

10. Dilnot A, Warner LN, Williams JJ. Fairer care funding. The report of the Commission on Funding of Care and Support. Dilnot Commission. https: / / www.wp.dh.gov.uk/carecommission/fi les/2011/07/Fairer-Care-Funding-Report.pdf.

11. Organization for Economic Co-operation and Development. Help wanted? Providing and paying for long-term care. Paris: OECD Publishing; 2011.

12. Maisonneuve C, Oliveira J. Public spending on health and long-term care: a new set of projections. OECD Economic Policy Papers 2013; 6:39.

13. Lipszyc B, Sail E, Xavier A. Long-term care: need, use and expenditure in the EU-27. Brussels: European Commission; 2012. (Economic Papers, 469).

14. Swartz K, Miake N, Farag N. Long-term care: common issues and unknowns. J Policy Anal Manage 2012; 31:139-52.

15. Swartz K. Searching for a balance of responsibilities: OECD countries' changing elderly assistance policies. Annu Rev Public Health 2013; 34:397-412.
16. Costa-Font J. Devolution, diversity and welfare reform: long-term care in the Latin rim? Soc Policy Adm 2010; 44:481-94.

17. Roit BD, Bihan BL. Similar and yet so different: cash-for-care in six European countries' long-term care policies. Milbank Q 2010; 88:286-309.

18. Kaye HS, Harrington C, LaPlante MP. Long-term care: who gets it, who provides it, who pays, and how much? Health Aff (Millwood) 2010; 29:11-21.

19. Fernandez J, Forder J. Reforming long-term care funding arrangements in England: international lessons. Appl Econ Perspect Policy 2012; 34: 346-62.

20. Comas-Herrera A, Wittenberg R, Pickard L. The long road to universalism? Recent developments in the financing of long-term care in England. Soc Policy Adm 2010; 44:391.

21. Colombo M. Help wanted? Fair and sustainable financing of long-term care services. Appl Econ Perspect Policy 2012; 34:316-32.

22. Campbell JC, Ikegami N, Gibson MJ. Lessons from public long-term care insurance in Germany and Japan. Health Aff (Millwood) 2010; 29:87-95.

23. Rostgaard T, coord. Livindhome: living independently at home: reforms in home care in 9 European countries. Copenhagen: Danish National Centre for Social Research; 2011.

24. Frank RG. Long-term care financing in the United States: sources and institutions. Appl Econ Perspect Policy 2012; 34:333-45.

25. Brown J, Finkelstein A. Insuring long-term care in the United States. J Econ Perspect 2011; 25:119-42.

26. Courbage C, Zweifel P. Two-sided intergenerational moral hazard, long-term care insurance, and nursing home use. J Risk Uncertain 2011; 43:65-80.

27. Barr N. Long-term care: a suitable case for social insurance. Soc Policy Adm 2010; 44:359-74.

28. Saraceno C, Keck W. Can we identify intergenerational policy regimes in Europe? European Societies 2010; 12:675-96.

29. Elosua P. Subjective values of quality of life dimensions in elderly people. A SEM preference model approach. Soc Indic Res 2011; 104:427-37.

30. Oien, Karlsson, Iversen. The impact of financial incentives on the composition of long-term care in Norway. Appl Econ Perspect Policy 2012; 34: 258-74.

31. Veras RP, Caldas CP, Motta LB, Lima KC, Siqueira $\mathrm{RC}$, Rodrigues RT, et al. Integration and continuity of care in health care network models for frail older adults. Rev Saúde Pública 2014; 48:357-65.

Recibido el 13/Mar/2015

Versión final presentada el 12/Sep/2015

Aprobado el 15/Oct/2015 